\title{
ldentificación de nichos de mercados en Ecuador para los programas educativos de la UNAD
}

\author{
Jesús Fandiño Isaza ${ }^{1}$
}

\begin{abstract}
Resumen
En el artículo se analiza la importancia que tiene la identificación de nichos de mercado para los programas educativos de la UNAD en Ecuador, a través del análisis de la evolución de los acuerdos comerciales, tratados firmados entre Colombia y Ecuador en materia educativa, los estudios realizados en Ecuador sobre población potencial de estudiantes, los sectores económicos y el contexto tecnológico de este país con relación a la educación superior. La investigación obtuvo resultados interesantes sobre diferentes universidades e instituciones de educación superior de Ecuador con las cuales existen condiciones para la definición de acuerdos, alianzas o consorcios con la UNAD, Colombia.
\end{abstract}

Palabras clave: nichos de mercados, Tratado de Libre Comercio (TLC), internacionalización educativa. 


\title{
Identifying market niches for UNAD's educational programs in Ecuador
}

\begin{abstract}
The article discusses the importance of identifying niche markets for the educational programs of the UNAD in Ecuador, through the analysis of the evolution of trade agreements, treaties between Colombia and Ecuador in education, such as studies Ecuador potential 0student population, the economic and technological context of this country in relation to higher education.

The investigation had interesting results on different universities and institutions of higher education in Ecuador with which conditions exist for the definition of agreements, alliances or consortia with UNAD, Colombia.
\end{abstract}

Keywords: Niche markets, Free Trade Agreement (NAFTA), Internationalizing Education.

Recibido: 16 de septiembre 2011

Aceptado: 25 de octubre 2011

\section{Introducción}

Los nichos de mercados son grupos más pequeños que los segmentos de mercados (Kotler, 2001) y se relacionan con mercados más reducidos cuyas necesidades no están bien atendidas, lo que convierte a este concepto en una herramienta fundamental para detectar necesidades o deseos específicos o parecidos con el mercado educativo de Colombia y en especial a los programas educativos de la UNAD. Es por esto que el fin del artículo es presentar un estudio sobre la importancia de la identificación de nichos de mercados en Ecuador para los programas educativos de la UNAD, a partir de una revisión documental sobre los principales elementos teóricos relacionados con los nichos de mercados.

Para lograr este fin, se realiza una revisión sobre la población potencial estudiantil, las apuestas productivas, los acuerdos comerciales para servicios educativos firmados entre Ecuador y Colombia, el contexto tecnológico de Ecuador y universidades o instituciones de educación superior con metodología similares a la UNAD. Los resultados están relacionados con cada uno de estos aspectos 
y en las conclusiones se presentan los nichos de mercados como estrategia fundamental para identificar los principales factores a tener en cuenta para la oferta de programas educativos en un país del exterior.

\section{Metodología}

El método de estudio es cuantitativo, con algunos análisis cualitativos, debido a que se trata de una revisión documental en fuentes secundarias sobre la identificación, descripción y análisis de los posibles programas educativos que por las características socioeconómicas y culturales de Ecuador, con respecto a Colombia, permitan que la oferta educativa de la UNAD pueda ser pertinente y admitida en ese país. Por tanto se requiere el desarrollo de una investigación de mercado que permita identificar los programas pertinentes de la oferta educativa de la UNAD en el Ecuador.

Las variables de estudio en la presente investigación son:

* Porcentaje de la población potencial estudiantil de educación superior del Ecuador.

* Número de apuestas productivas del Ecuador que permitan sugerir la oferta educativa de la UNAD.

* Número de acuerdos comerciales de servicios educativos firmados en tratados entre Ecuador y Colombia

* Contexto tecnológico del Ecuador en la Educación Superior.

* Cantidad de universidades o instituciones de educación superior con metodología similares a la UNAD el en Ecuador que permitan definir convenios, alianzas o consorcios viables.

De acuerdo con la investigación documental realizada, se analizaron las fuentes secundarias necesarias para llevar a cabo el trabajo y entre las que se pueden citar diversos tipos de documentos:

* Documentos secundarios: boletines de resúmenes (analíticos, indicativos, índices de citas), bibliografías, repertorios y directorios. Se han utilizado de acuerdo con información seleccionada de sitios web, como se describirá en el siguiente punto.

* Documentación informatizada: Internet, catálogos en línea, buscadores, bases de datos especializadas, etc. Estas son las fuentes al momento 
consultadas, dentro de las que se destacan las siguientes páginas del Ecuador: página del instituto nacional de estadísticas y censos del Ecuador, sitio web del Instituto Internacional para la Educación Superior en América Latina y el Caribe (Iesalc) que es un organismo de la Organización de las Naciones Unidas para la Educación, la Ciencia y la Cultura (Unesco), dedicado a la promoción de la educación superior, contribuyendo a implementar en la región latinoamericana y caribeña el programa que, en materia de educación superior, página del Consejo Nacional de Educación del Ecuador y la página del Ministerio de Educación del Ecuador.

* Se han analizado un total de 5 investigaciones relacionadas con la Educación Superior de aquel país y 16 proyectos de investigación sobre nuevos proveedores de educación superior en otros países de América, algunos relacionan las URL de internet con la respectiva fecha cuando se consultaron. Cuatro (4) sitios web mencionados anteriormente, relacionadas con este país.

\section{Resultados y discusión}

Los resultados indican que las apuestas productivas de Ecuador tienen una relación directa con la oferta de los programas educativos de la UNAD, entre los cuales se destacan la agricultura, industria manufacturera, servicios públicos, construcción, comercio y hoteles, transporte y comunicación y los servicios financieros. En relación a la población estudiantil del Ecuador en el año 2006, esta era de 312.809, según datos del Consejo Nacional de Educación Superior (Conesup), de los cuáles el 69,43\%, corresponden a 217.208 estudiantes que están en universidades y politécnicas públicas. El sector privado con cofinanciación cuenta con 82.715 estudiantes, y en el régimen auto financiado 60.316 estudiantes.

La población educativa de carácter superior está distribuida, según el Conesup de la siguiente manera, 76,6\% en entidades públicas y el $23.4 \%$ en privadas. Las carreras con mayor población son: Administración y Comercio $30.38 \%$, Ciencias de la Salud 20.57\%; Ciencias Sociales 15,71\% y poco más de $14 \%$ para los programas de pregrado en Educación. Los programas tecnológicos solo representan un $8.77 \%$.

Tabla 1. Informe de educación superior por áreas en Ecuador, en el 2006

\begin{tabular}{|c|c|}
\hline Áreas & Proporción \\
\hline Administración y Comercio & $30.38 \%$ \\
Agrícola y Pecuaria & $4.86 \%$ \\
Artes y Arquitectura & $0.87 \%$ \\
\hline
\end{tabular}




\begin{tabular}{|c|c|}
\hline Áreas & Proporción \\
\hline Ciencias Básicas & $3.47 \%$ \\
Ciencias de la Salud & $20.57 \%$ \\
Ciencias Sociales & $15.71 \%$ \\
Educación & $14.84 \%$ \\
Humanidades y Ciencia de la Cultura & $0.52 \%$ \\
\hline Tecnología & $8.77 \%$ \\
\hline
\end{tabular}

Fuente: Archivos del Consejo Nacional de Educación Superior (Conesup)

Tabla 2. Informe de Institutos Superiores Técnicos y Tecnológicos por áreas en Ecuador, en el 2006.

\begin{tabular}{|c|c|}
\hline Áreas & Proporción \\
\hline Administración y Comercio & $40.72 \%$ \\
Agrícola y Pecuaria & $5.87 \%$ \\
Artes y Arquitectura & $9.86 \%$ \\
Ciencias Básicas & $0.47 \%$ \\
Ciencias de la Salud & $1.35 \%$ \\
Ciencias Sociales & $4.46 \%$ \\
Educación & $6.28 \%$ \\
Humanidades y Ciencia de la Cultura & $0.74 \%$ \\
\hline Tecnología & $30.25 \%$ \\
\hline
\end{tabular}

Fuente: Archivos del Consejo Nacional de Educación Superior (Conesup)

En la demanda de los programas de Institutos Superiores Técnicos y Tecnológicos indica que estos cubren el 30, 25\% y los programas que se destacan son Administración y Comercio 40.72\%, y las carreras de Artes y Arquitectura tienen el 10\%.

El sistema educativo preprimaria, primario y medio ecuatoriano, cuenta con una población de 3.931.637 estudiantes entre los años 2009 - 2010, según datos publicados por el Ministerio de Educación y Cultura. Los valores absolutos relacionados con números de estudiantes en el sistema (fiscal y privado), aparecen en la tabla 3 .

Tabla 3. Alumnos por nivel educativo y régimen escolar AMIE 2009-2010, según la ubicación geográfica a nivel nacional.

\begin{tabular}{|c|c|c|c|}
\hline \multirow{2}{*}{ Niveles } & \multicolumn{3}{|c|}{ Régimen } \\
\cline { 2 - 4 } & Costa & Sierra & Total \\
\hline Bachillerato & 14.556 & 2.097 & 16.653 \\
\hline EGB & 1.182 .243 & 784.986 & 1.967 .229 \\
\hline
\end{tabular}


Identificación de nichos de mercados en Ecuador para los programas educativos de la UNAD, artículo

\begin{tabular}{|c|c|c|c|}
\hline \multirow{2}{*}{ Niveles } & \multicolumn{3}{|c|}{ Régimen } \\
\cline { 2 - 4 } & Costa & Sierra & Total \\
\hline Bachillerato & & & 1.340 .412 \\
\hline EGB y Bachillerato & 673.810 & 666.602 & 37.143 \\
\hline Inicial & 17.872 & 19.271 & 57 \\
\hline Inicial y Bachillerato & 38 & 19 & 394.943 \\
\hline Inicial y EGB & 239.908 & 155.035 & 175.200 \\
\hline Inicial, EGB y & 55.463 & 119.737 & 3.931 .637 \\
\hline Bachillerato & & & \\
\hline Total & 2.183 .890 & 1.747 .747 & \\
\hline
\end{tabular}

Fuente: Ministerio de Educación Nacional de Educador.

El sistema universitario ecuatoriano tiene una oferta de variedad de programas afines con los sectores económicos, relacionados en la tabla 4. Los 10 sectores económicos producen el $72 \%$ del empleo por su incidencia directa en el PIB, por el aporte importante a la oferta exportadora del Ecuador 2009, relacionados en la tabla 4.

Tabla 4. Sectores Económicos de Ecuador 1998.

\begin{tabular}{|c|}
\hline Sectores \\
\hline Agricultura, caza, silvicultura y pesca \\
Petróleo y minas Industria Manufactura Electricidad, gas y agua \\
Construcción Comercio y hoteles Transporte y comunicación \\
Servicios financieros
\end{tabular}

Fuente: SICA - MAG.

La relación de la economía con los programas educativos que ofertan las instituciones de educación superior en el Ecuador, se resumen en la tabla No 2, y la tabla No 5 sobre la proporción de personal empleado por actividad del año 2000 al 2005 , debido a que hay una alta proporción de pertinencia de los programas con el personal empleado por actividad económica. No obstante, otras actividades presentan dinámicas de crecimiento importantes como restaurantes y hotelería; agricultura y ganadería; electricidad y agua potable, mientras que la pesca y la minería tienen un menor ritmo de crecimiento, categorizadas como sectores no generadores de empleo.

Tabla 5. Proporción de población económicamente activa del 2000 al 2005 según rama de actividad.

\begin{tabular}{|l|l|l|l|l|l|l|}
\hline Rama de actividad & $\mathbf{2 0 0 0}$ & $\mathbf{2 0 0 1}$ & $\mathbf{2 0 0 2}$ & $\mathbf{2 0 0 3}$ & $\mathbf{2 0 0 4}$ & $\mathbf{2 0 0 5}$ \\
\hline Agricultura, ganadería y caza & $7,0 \%$ & $6,5 \%$ & $7,5 \%$ & $7,8 \%$ & $8,3 \%$ & $6,6 \%$ \\
\hline Pesca y criaderos & $1,5 \%$ & $1,2 \%$ & $1,0 \%$ & $1,3 \%$ & $1,0 \%$ & $1,0 \%$ \\
\hline
\end{tabular}




\begin{tabular}{|c|c|c|c|c|c|c|}
\hline Rama de actividad & 2000 & 2001 & 2002 & 2003 & 2004 & 2005 \\
\hline Explotación de minas y canteras & $0,6 \%$ & $0,5 \%$ & $0,6 \%$ & $0,6 \%$ & $0,4 \%$ & $0,4 \%$ \\
\hline Industria Manufacturera & $15,6 \%$ & $16,6 \%$ & $14,5 \%$ & $13,8 \%$ & $14,0 \%$ & $14,2 \%$ \\
\hline Suministro de electricidad gas y agua & $0,6 \%$ & $0,8 \%$ & $0,4 \%$ & $0,5 \%$ & $0,6 \%$ & $0,6 \%$ \\
\hline Construcción & $7,1 \%$ & $6,4 \%$ & $7,0 \%$ & $6,8 \%$ & $6,4 \%$ & $5,7 \%$ \\
\hline \multicolumn{7}{|l|}{ Comercio, y Reparación de vehículos } \\
\hline Efectos personales & $26,6 \%$ & $28,0 \%$ & $28,1 \%$ & $28,3 \%$ & $28,4 \%$ & $29,0 \%$ \\
\hline Hoteles y restaurantes & $4,3 \%$ & $4,3 \%$ & $4,2 \%$ & $3,7 \%$ & $4,4 \%$ & $5,4 \%$ \\
\hline $\begin{array}{l}\text { Transporte almacenamiento } \\
\text { y comunicación }\end{array}$ & $6,3 \%$ & $6,7 \%$ & $6,4 \%$ & $6,6 \%$ & $6,9 \%$ & $7,0 \%$ \\
\hline Intermediación financiera & $1,3 \%$ & $0,9 \%$ & $1,3 \%$ & $1,5 \%$ & $1,3 \%$ & $1,2 \%$ \\
\hline $\begin{array}{l}\text { Act. Inmobiliarias, empresariales } \\
\text { y alquiler }\end{array}$ & $4,0 \%$ & $4,3 \%$ & $4,5 \%$ & $4,4 \%$ & $4,9 \%$ & $4,8 \%$ \\
\hline $\begin{array}{l}\text { Admón. pública y defensa, } \\
\text { seguridad social }\end{array}$ & $4,1 \%$ & $4,4 \%$ & $4,2 \%$ & $5,1 \%$ & $4,5 \%$ & $4,7 \%$ \\
\hline Enseñanza & $6,6 \%$ & $5,8 \%$ & $6,9 \%$ & $6,6 \%$ & $6,8 \%$ & $7,4 \%$ \\
\hline Act. servicios sociales y de salud & $3,2 \%$ & $2,7 \%$ & $3,4 \%$ & $3,3 \%$ & $3,6 \%$ & $3,2 \%$ \\
\hline $\begin{array}{l}\text { Otras actividades comunitarias } \\
\text { sociales y personales }\end{array}$ & $4,9 \%$ & $4,2 \%$ & $3,5 \%$ & $4,9 \%$ & $4,6 \%$ & $4,5 \%$ \\
\hline $\begin{array}{l}\text { Hogares privados con servicio } \\
\text { Doméstico }\end{array}$ & $6,4 \%$ & $6,3 \%$ & $6,2 \%$ & $4,6 \%$ & $3,9 \%$ & $4,4 \%$ \\
\hline Organizaciones Extraterritoriales & $0,0 \%$ & $0,0 \%$ & $0,0 \%$ & $0,0 \%$ & $0,0 \%$ & $0,0 \%$ \\
\hline No especifica & & $0,5 \%$ & $0,0 \%$ & $0,0 \%$ & $0,0 \%$ & $0,0 \%$ \\
\hline
\end{tabular}

Fuente: Instituto Nacional de Estadísticas y Censos -INEC-. (SF).

Otros resultados se dan con la movilidad ocupacional frente a las ramas de actividad por grupos ocupacionales, donde se destaca el personal directivo, perteneciente al sector público y privado, con un repunte al año 2004. Sin embargo se analiza una baja en el grupo de formados a nivel directivo y de empleados de oficina; en cambio en el caso de personal técnico se observa un constante ritmo de crecimiento de la ocupación en el área urbana. 


\section{Jesús Fandiño Isaza}

Identificación de nichos de mercados en Ecuador para los programas educativos de la UNAD, artículo producto de la investigación

Figura 1. Índice de ocupación personal empleado por actividad del 2000 al 2005, área urbana de Ecuador

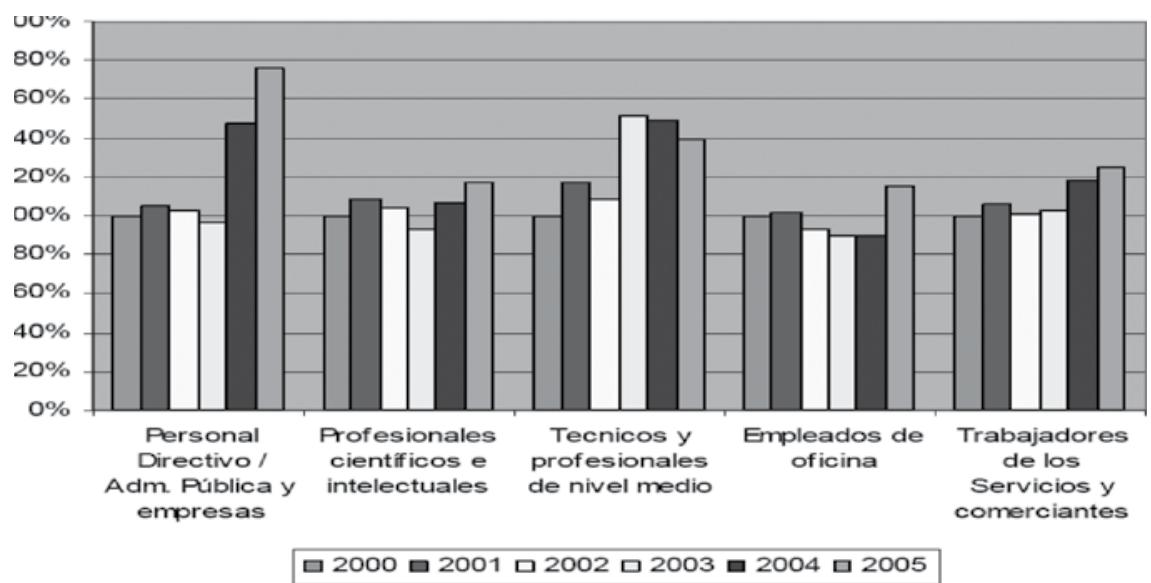

Fuente: Análisis y proyección de la población económicamente activa (PEA) del Ecuador-INEC.

Figura 2. Índice de ocupación de personas empleadas por actividad en el área urbana de Ecuador del 2000 al 2005, según grupo de ocupación.

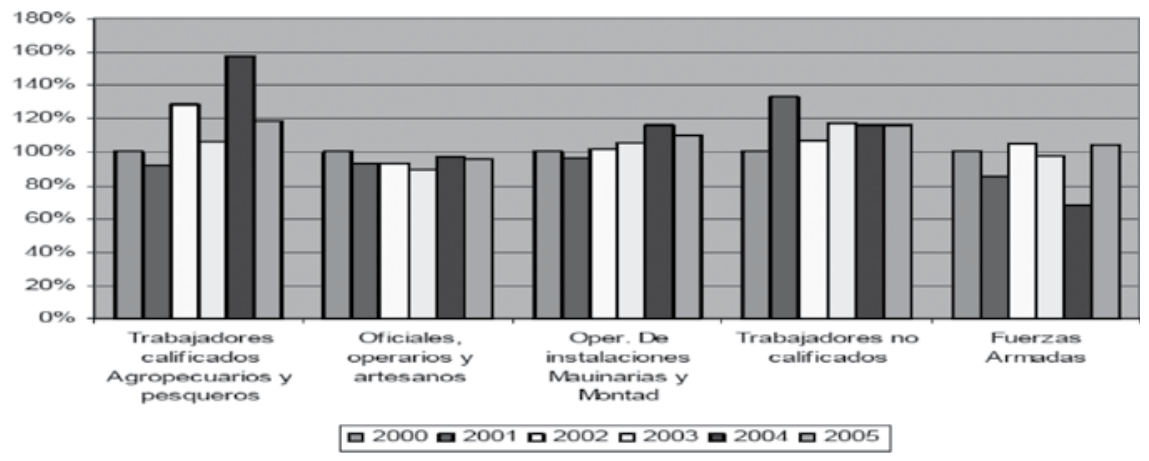

Fuente: análisis y proyección de la población económicamente activa (PEA) del Ecuador-INEC

Acuerdos de comercialización de servicios educativos contenidos en tratados internacionales relacionados con Ecuador y Colombia.

Dentro de los acuerdos se resalta el Convenio Andrés Bello, iniciado en 1970 en Venezuela, que agrupa los siguientes países: Bolivia, Chile, Colombia, Ecuador, España, México, Panamá, Perú, Paraguay y Venezuela. La alianza de Universidades de América Latina (UDUAL) fundada en 1949; la Organización 
Universitaria Iberoamericana (OUI) creada en 1980. La Organización de Estados Iberoamericanos (OEI) que dirige desde los años 2000-2001 el Programa de Intercambio y de Movilidad Académica entre España, Argentina, Brasil, Bolivia, Colombia, Costa-Rica, Cuba, Chile, Ecuador, México, Nicaragua, El Salvador, Panamá, Perú, Uruguay y Venezuela. El Consejo Universitario Iberoamericano, fundado en 2002 en Colombia que convoca organizaciones universitarias de América Latina, España y Portugal para intercambiar experiencias y favorecer iniciativas conjuntas de integración regional.

Otro tratado es la Asociación Latinoamericana de Integración (ALADI), firmada en Montevideo (1980), cuyo propósito es el establecimiento de forma gradual y progresiva de un mercado común latinoamericano. Integrada por países como Argentina, Bolivia, Brasil, Chile, Colombia, Ecuador, México, Paraguay, Perú, Uruguay y Venezuela. Este tratado tiene un alcance regional para acciones de cooperación en materia científica y tecnológica, de cooperación e intercambio de bienes en las áreas cultural, educacional y científica y de superación de obstáculos técnicos al comercio. En lo relacionado a la cooperación e intercambio de bienes en las áreas culturales, educacional y científica se busca propender por un extenso marco de cooperación educativa, cultural y científica de los países asociados y a mejorar y elevar los niveles de instrucción, capacitación y conocimiento recíproco de los pueblos de la región.

El acuerdo del Grupo de Rio para la cooperación educativa creado en 1988, que dentro de sus campos de acción, tiene el promover la formación progresiva de un mercado tradicional de bienes culturales y educativos, que facilite la libre circulación en los territorios de los países miembros y transitoriamente a los demás países latinoamericanos y del Caribe. Se destacan los Estados Asociados y Parte del Mercado Común del Sur (Mercosur), que suscribieron los siguientes acuerdos en materia educativa y en los cuales Ecuador adquiere una responsabilidad con la integración regional, mediante la incorporación de este país al Protocolo de Integración Educativa y Reconocimiento de Certificados, Títulos y Estudios de Nivel Primario y Medio no Técnico entre los países integrantes del Mercosur y la firma del memorando de alcance sobre instauración y puesta en funcionamiento de un sistema de acreditación de carreras universitarias para el reconocimiento regional de la calidad académica, relacionados con las titulaciones que se den a través del Mercosur y países asociados, documento que permite la movilidad de personas entre los países de la región y de aval a los mecanismos regionales de reconocimiento de títulos y diplomas universitarios (Mercosur Educativo, 2009).

De otra parte la Unesco firmó un acuerdo similar sobre reconocimientos de títulos y diplomas, a través del Comité Regional de América Latina y el Caribe (CRALC) mediante el convenio regional de convalidación de Estudios, Títulos y Diplomas de educación superior para América Latina y el Caribe, firmado por 18 países, de los cuales 13 pertenecen a la región (entre los que se resaltan Colombia y Ecuador) y otros cinco son de otras zonas geográficas. 
Identificación de nichos de mercados en Ecuador para los programas educativos de la UNAD, artículo

\section{Entorno tecnológico de Ecuador relacionado con la educación superior}

La suscripción de acuerdos para la libre oferta de servicios educativos, requiere de los países asociados, contar con una infraestructura tecnológica y de conectividad adecuada para la exportación de programas educativos a los países agrupados en el tratado. Frente a este requerimiento Ecuador presenta una infraestructura tecnológica y de conectividad con el índice más bajo de avance de Internet América Latina, no obstante en los últimos tres años, tanto la oferta como la demanda se ha elevado de forma sostenida, provocada por el fenómeno global de la incorporación y utilización del Internet en las empresas y universidades ecuatorianas que en los últimos años han digitalizado gran parte de su infraestructura (Unesco, 2004).

Los servicios de acceso a Internet de las universidades en Ecuador son aceptables, no obstante un porcentaje significativo no contesta (32\%) y se podría suponer que no poseen este servicio. El 68\% cuenta con conexiones que van desde los $64 \mathrm{Kbps}$ hasta 1,5 Mbps, dos (02) universidades ofrecen servicios de ISP (proveedores de acceso a Internet) y cuentan con una cartera de clientes importantes (Unesco, 2004).

En Ecuador las universidades con acceso a Internet cuentan con páginas web y correo electrónico, respaldadas por sus correspondientes servidores, la cantidad de estos es de 1 a 5 , teniendo en cuenta el número de estudiantes que tengan. En referencia a la infraestructura interna, el $52 \%$ cuenta con fibra óptica, especialmente las universidades que cuentan con un mayor número de estudiantes y por lo tanto la carga de trabajo en sus redes internas es mayor (Unesco, 2004).

Con respecto al uso de recursos virtuales para inscripción, administración, pagos, video conferencia, biblioteca virtual, campus virtual, el $72 \%$ de las universidades ecuatorianas no utilizan estos recursos y el $28 \%$ si lo hace. De igual forma la incorporación de tecnologías en el desarrollo de programas académicos inició en 1999. En el año 2001 había programas a distancia y semipresenciales con apoyo de Internet ofertados por la Universidad Técnica Particular de Loja (UTPL) que consistían en 3 diplomados con apoyo de tecnología, que incluía un módulo a distancia y el componente de videoconferencia que llega a 23 ciudades, finalmente este proceso se fortaleció a partir del año 2002 porque se contaba con ofertas de formación continuada y de pregrado completamente en línea (Unesco, 2004).

En estos mismos años 2001 y 2002, otras universidades ofertaron carreras de pregrado a distancia con apoyo del Internet como son la Escuela Superior Politécnica del Ejército (ESPE), la Universidad de las Américas (UDLA) y la Universidad San Francisco de Quito (USFQ), que se destaca porque cuenta con ocho carreras mediadas por tecnologías, cada una se forma de cursos y cada 
uno de estos requiere de una carga de trabajo de 45 horas, de las cuales 9 son presenciales al igual que las evaluaciones (Unesco, 2004).

Para el caso de programas académicos totalmente en línea, en el año 1999, la Universidad Técnica Particular de Loja (UTPL) lanzó un curso de «Lectura Comprensiva de Inglés para Médicos» en una plataforma montada sobre Lotus Notes, el cual tuvo poca acogida y una duración de dos meses. La misma UTPL en el año 2002, ofertó un total de 30 cursos y un programa universitario a través de campus virtual (Unesco, 2004).

Un aspecto positivo en Ecuador es la posibilidad que tienen las universidades para ofertar programas a distancia, debido a que el porcentaje de las mismas que cuentan con este tipo de programas es muy representativo, lo que marca una tendencia y crea competencia entre las universidades, que implica que realicen mejoras de calidad en los programas y en los recursos que se utilizan. Para el año 2002 en Ecuador el 72\% de las universidades tenían programas presenciales y a distancia, el $24 \%$ presencial y $4 \%$ semi- presencial, lo que representa un dato muy importante debido a que estas universidades apoyarán cada vez más con tecnología sus programas académicos a distancia tradicionales (Unesco, 2004).

Se destaca la información arrojada en la última encuesta de Tecnologías de la Información y Comunicaciones TIC'S, realizada por el Instituto Nacional de Estadística y Censos (INEC), en Ecuador en el año 2008 a 20.220 hogares, divididas en 11.328 viviendas urbanas y 8.892 rurales (las personas encuestadas eran de cinco años en adelante). El resumen de los resultados son los siguientes:

* El $41 \%$ de los ecuatorianos ha utilizado computadoras alguna vez en su vida, $51 \%$ del área urbana y $20,9 \%$ de la rural.

* El uso de internet en el hogar es del 21,6\% mientras que en el área urbana es del $23,5 \%$ y en el área rural 7,2\%. En el trabajo el uso es del $14,4 \%$ a nivel nacional, en el área urbana el 15,3\% y el área rural el 7,6\%.

* Los individuos que han usado alguna vez en su vida el internet, desde cualquier lugar a nivel nacional son de $26 \%$, siendo $34 \%$ en el área urbana y $9 \%$ en el área rural.

* Otros resultados que arroja el estudio dice que el 25,3\% de la gente ha utilizado Internet alguna vez en su vida, el $38,9 \%$ lo ha hecho en centros de acceso público como cibercafés, mientras el $21,6 \%$ en el hogar y el $14,4 \%$ en el trabajo.

* El 45,5\% de las veces, los encuestados se conecta para actividades de educación y aprendizaje y el 7,3\% lo hace por razones de trabajo. Así el 
$40,1 \%$ utiliza la Internet por lo menos una vez a la semana, mientras el $37,3 \%$ lo hace una vez al día.

* El uso de internet en el hogar es del 21,6\% mientras que en el área urbana es del $23,5 \%$ y en el área rural 7,2\%. En el trabajo el uso es del $14,4 \%$ a nivel nacional, en el área urbana el 15,3\% y el área rural el 7,6\%.

* El 40,1\% de la veces se utiliza internet para educación y aprendizaje, el $23,9 \%$ para comunicación y el 7,3\% para trabajo.

* Los hogares que poseen acceso a internet a nivel nacional son el 7\%, siendo $10 \%$ en el área urbana y $1 \%$ en el área rural.

* De los hogares encuestados, el 7\% tiene acceso a Internet, el 43,4\% lo tiene a través de módem o teléfono, el $52,3 \%$ por cable o banda ancha y el 3,4\% posee Internet inalámbrico.

La cultura de uso de Internet y su tasa de penetración han incidido en el lento desarrollo de la educación a distancia mediada por tecnologías en Ecuador, sin embargo la oferta de programas de prestigiosas universidades internacionales obliga a la universidad ecuatoriana a repensar su accionar y a establecer alianzas académicas que le permitan aprovechar su experiencia e infraestructura, como la UNAD.

Universidades o instituciones de educación superior con metodología similares a la $U N A D$ en el Ecuador que permitan definir convenios, alianzas o consorcios viables

En Ecuador existe un total de 67 universidades y 8 escuelas politécnicas reconocidas por el estado ecuatoriano, igualmente 380 «institutos superiores técnicos y tecnológicos» que también se amparan en la Ley de Educación Superior e integran el Sistema de Educación Superior. De estas universidades el $27 \%$, prestan servicios a través de la modalidad a distancia - on line, y el $73 \%$ de forma presencial, y algunas de ellas, pueden mezclar la modalidad semi-presencial. De esta forma se tiene que existen 19 universidades, t3 de ellas con registro nacional de acreditación, con las cuáles se pueden realizar al menos un convenio, alianza o consorcio, ya que pueden facilitar la oferta de los programas de la UNAD en Ecuador, de acuerdo con datos del Conesup (2009).

Estas alianzas o convenios se pueden dar, debido a que en Ecuador las universidades trabajan sus procesos académicos mediante la unidad de crédito académico que distribuye entre la población por nivel Técnico Superior $8 \%$, Pregrado (Tercer nivel) 89,5\% y Postgrado 2.5\%. El número de créditos para optar al titulo se ordenan en Técnico 120, Tecnólogo 185 y Pregrado completo 240 créditos. Los créditos académicos de diploma superior 15, para Especialista 30, Magister 60 y Doctorado 90, según el Conesup (2006). 
La cantidad de créditos en Ecuador no son equivalentes a los establecidos por la UNAD de Colombia, por ejemplo para la carrera de psicología con 162 créditos, comunicación 160 y filosofía 160, están por debajo de los 240 créditos exigidos en este país. De igual forma, los institutos técnicos y tecnológicos presentan, en sus correspondientes áreas, un total de 1.008 ofertas profesionales, otorgando, según la ley correspondiente, «títulos profesionales de técnico, tecnólogo o ingeniero de operación» (Conesup, 2006).

En Ecuador se privilegia la oferta de carreras en administración y comercio, lo que se combina con factores de cada vez mayor peso del sector servicio en la economía nacional, el aumento en el uso de las Tics en la vida social, el permanente consumismo y la relativa baja inversión que requiere ofertar este tipo de carreras (Conesup, 2006).

En conclusión en Ecuador existen programas acreditados, con los cuales cuenta la UNAD y que puede llegar a ofertar como Agronomía, Ingeniera Industrial e Ingeniería Electrónica, los cuales tienen reconocimiento en muchos países miembros y asociados de estos tratados, como Colombia y Ecuador.

\section{Conclusiones}

La población educativa del Ecuador en un $76,6 \%$ prefieren entidades públicas y el $23.4 \%$ privadas.

La totalidad de los estudiantes de institutos superiores técnicos y tecnológicos en Ecuador optan por programas análogos a los ofertados a la UNAD, como Administración y Comercio en un $40.72 \%$ y de Tecnologías en un 30.25\%.

En Ecuador los sectores productivos, de industria manufacturera, agricultura, comercio, construcción, transporte y enseñanza se pueden considerar nichos de mercados potenciales para los programas educativos de la UNAD.

Se pueden considerar otros nichos de mercados potenciales los sectores productivos de restaurantes y hotelería; agricultura y ganadería; electricidad y agua potable por ser importantes generadoras de empleo en el Ecuador.

En la investigación se destacan siete (7) tratados o acuerdos comerciales internacionales para oferta y movilidad de programas educativos entre Ecuador y Colombia, ellos son: Andrés Bello, UDUAL, OUI, OEI, El Consejo Universitario Iberoamericano, constituido en 2002 en Colombia, el ALADI y el Mercosur.

La infraestructura de acceso a Internet de las universidades es de un $68 \%$ y que cuenta con enlaces que van desde los $64 \mathrm{Kbps}$ hasta 1,5 Mbps. Además las 
Identificación de nichos de mercados en Ecuador para los programas educativos de la UNAD, artículo

universidades con acceso a Internet cuentan con servidores y correo electrónico, lo que garantiza su presencia en la red con sus respectivas páginas web.

En Ecuador hay experiencia de oferta de programas de diplomado con apoyo de tecnología de tipo semipresenciales, con un componente a distancia y con un componente de videoconferencias por medio de la red de videoconferencia satelital.

En Ecuador existen 19 universidades on-line, que equivalen al 28\% de las universidades y el $72 \%$ son presenciales, en las que algunas pueden mezclar la modalidad semi-presencial para ofertar sus programas.

\section{Referencias bibliográficas}

Asociación Nacional de Universidades e Instituciones de Educación Superior (ANUIES) - Unesco. 2004. La Educación Superior Virtual en América Latina y el Caribe. México. Consultado el 11 de noviembre de 2009 en: http://books.google.com.co/books?id=Z hogX8OodyoC\&lpg=PP1\&dq=inauthor\%3 A\%22Anuies-unesco\%22\&hl=es\&pg=PA6\#v= onepage $\& q \& f=$ false

Instituto Nacional de Estadísticas y Censos -INEC-. (s.f.). «Estudios Demográficos en Profundidad». Análisis y Proyección de la Población Económicamente Activa (PEA) del Ecuador. Consultado el 10 de Noviembre de 2009 en: http://www.inec.gov.ec/web/ guest/publicaciones/estudios/soc/dem_prof

IESALC. 2003. Internacionalización y nuevos proveedores de educación superior en Ecuador. Consultado el 10 de noviembre de 2009 en: http://unesdoc.unesco.org/ images/0014/001404/140479s.pdf

IESALC. 2005. «Propuesta de modificación del Convenio Regional de Convalidación de Estudios, Títulos y Diplomas de Educación Superior en América Latina y El Caribe. 1974 y Borrador de propuestas y recomendaciones formuladas al tenor de la Reunión Convenio Regional de Convalidación de Estudios, Títulos y Diplomas de Educación Superior en América Latina y El Caribe, realizada en Bogotá». Consultado el 10 de noviembre de 2009 en: http://iesalc.unesco.org.ve/pruebaobservatorio/ documentos pdf/.

Kotler, P. 2001. Dirección de Marketing, La Edición del Milenio. México: Pearson, Prentice Hall.

Mercosur Educativo. 2009. Portal del Sector Educativo. Consultado el 12 de noviembre de 2009 en: http://www.sic.inep.gov.br/. 


\section{Revista de}

investigaciones UNAD

Volumen 10. Número 2. Diciembre 2011

Sistema de Educación Superior del Ecuador. 2006. Consejo Nacional de Educación Superior (Conesup). Consultado el 12 de noviembre de 2009 en: http://tuning. unideusto.org/tuningal/images/stories/presentaciones/ecuador.pdf 